\title{
Changes in coagulation functions and hemorheological parameters may predict hematoma formation after total knee arthroplasty
}

Ning Liu ${ }^{1,2+}$, Simin Luo ${ }^{1,2+}$, Cheanglek Hang ${ }^{3+}$, Zhengang Zha ${ }^{1,2^{*}}$, Jieruo $\mathrm{Li}^{1,2}$, Wenrui $\mathrm{Wu}^{1,2}$ and Dabiao Hou ${ }^{1,2}$

\begin{abstract}
Background: Hematoma formation around the knee is commonly seen after total knee arthroplasty (TKA) and may cause patient discomfort and worry regarding the success of the surgery. This study aimed to evaluate the coagulation functions and hemorheological parameters in patients undergoing TKA and investigate their associations with hematoma formation.

Methods: This study prospectively included 146 patients treated for knee osteoarthritis by unilateral TKA between August 2013 and August 2014. Apixaban was administered twice during the 12-24-h period after surgery. Blood coagulation functions were evaluated according to activated partial thromboplastin time (APTT), prothrombin time (PT), thrombin time, and fibrinogen preoperatively and on postoperative days 1, 3, 7, and 14. Hemorheological parameters were also measured. Patients were divided into a hematoma group and a non-hematoma group for comparison.

Results: On postoperative day 1, the hematoma group showed significantly prolonged APTT and PT and significantly decreased hematocrit relative to baseline values $(P<0.05)$. The whole blood high shear rate, whole blood low shear rate, plasma viscosity, and hematocrit did not differ significantly between the two groups at baseline or from postoperative days $1-14$ in $(P>0.05)$.

Conclusions: Prolonged APTT and PT on the first day after TKA as well as decreased hematocrit may indicate an increased risk of hematoma formation. Postoperative use of apixaban may promote the formation of ecchymoses but is not a major contributing factor.
\end{abstract}

Keywords: Activated partial thromboplastin time, Prothrombin time, Total knee arthroplasty, Hematoma, Apixaban

\section{Background}

Total knee arthroplasty (TKA) is an effective treatment for advanced pathological conditions of the knee. TKA is performed increasingly often due to the global aging trend. However, this procedure is associated with some complications. Patients undergoing TKA are often at a hypercoagulable state and prone to the development of thrombosis. If not properly managed, the incidence of venous thromboembolism (VTE) in these patients can

\footnotetext{
* Correspondence: zhzgg@vip.163.com

${ }^{\dagger}$ Equal contributors

'The First Affiliated Hospital, Jinan University, Guangzhou 510632, China ${ }^{2}$ Institute of Orthopaedic Disease Research, Jinan University, Guangzhou 510632, China

Full list of author information is available at the end of the article
}

be as high as $40-80 \%$, resulting in a mortality rate of $2 \%$ due to symptomatic pulmonary embolism $[1,2]$.

The American Academy of Orthopaedic Surgeons and the American College of Chest Physicians developed new evidence-based guidelines for VTE prophylaxis after total joint arthroplasty in 2013. According to the updated guidelines, one of the following agents should be used for a minimum of 14 days after surgery: warfarin, low-molecular-weight heparin, fondaparinux, aspirin, rivaroxaban, dabigatran, apixaban, or portable mechanical compression. Moreover, bleeding tendencies in these patients must be monitored carefully [2]. Although these prophylaxis treatments very rarely cause major hemorrhaging, their use during the first 2 weeks after TKA 
more commonly contributes to the occurrence of ecchymoses and limb swelling, complications that may cause worry among patients and delay the initiation of exercise, leading to compromised clinical outcomes [3, 4].

Many factors have been proposed to be related to hematoma formation after TKA, such as the concurrent use of continuous femoral nerve block and an anticoagulant [3], postoperative drainage patterns [4-7], tourniquet use during operation $[8,9]$, postoperative anticoagulant use [10], and postoperative lower limb positioning [11, 12]. However, the specific underlying reasons for hematoma formation after TKA remain unclear. We hypothesized that hypercoagulability and hemorheological changes are involved in the development of ecchymoses after TKA. To test this hypothesis, in this study, we evaluated the coagulation functions and hemorheological parameters during the perioperative period of TKA and analyzed potential associations with hematoma formation.

\section{Methods \\ Patients}

This study included 146 patients with knee osteoarthritis who were treated between August 2013 and August 2014 at our hospital. The patients included 51 males and 95 females and had a mean age of 70 years (range, 6478 years). Osteoarthritis was diagnosed according to the criteria provided by the American College of Rheumatology in 1995 [13]. Only patients with surgical indications for TKA were included in this study. The surgical indications for TKA were several instability, pain, deformity, and dysfunction of the knee joint. All patients underwent unilateral TKA. Exclusion criteria were the patients with end-stage renal disease, severe liver dysfunction, major comorbidities (bleeding disorders, ischemic heart disease, and peripheral vascular diseases), and any kind of cancer disease. Informed written consent was obtained from all patients, and the study was approved by the Ethics Committee of the First Affiliated Hospital, Jinan University.

\section{Postoperative management}

Drainage tubes were placed postoperatively and removed within 24-48 h. The tubes were not temporarily clipped after TKA. Antibiotics were used for 3 days to prevent infection. Patients were instructed to wear elastic stockings. Apixaban at a dose of $2.5 \mathrm{mg}$ was administered twice during the $12-24-\mathrm{h}$ period postoperatively. Patients were encouraged to initiate exercise from the first day after surgery as a prophylaxis measure for deep vein thrombosis. Patients were closely monitored to record the occurrence of hematoma and divided into a hematoma group and a non-hematoma group. All patients were followed up for at least 2 months, and no cases of symptomatic VTE were observed.

\section{Blood measurements}

Peripheral blood was collected from each patient preoperatively and on postoperative days $1,3,7$, and 14 for blood measurements. Blood coagulation functions were evaluated based on activated partial thromboplastin time (APTT), prothrombin time (PT), thrombin time (TT), and fibrinogen (FIB) using an automated blood coagulation analyzer (STAGO, France). Hemorheological parameters were measured using a hemorheological analyzer (LBY-N6C, Puli Inc., Beijing, China).

\section{Statistical analysis}

Continuous data are presented as mean \pm standard deviation values and were compared using Student $t$ tests or one-way analysis of variance. Categorical data are presented as frequencies or percentages and were compared using $X^{2}$ tests. All statistical analyses were performed using SPSS 16.0 software for Windows (SPSS Inc., Chicago, IL, USA). $P<0.05$ was considered statistically significant.

\section{Results}

\section{Patient information}

No significant differences were found in terms of sex, age, body mass index (BMI), and presence of diabetes or hypertension between patients in the hematoma group $(n=32)$ and those in the non-hematoma group $(n=114$; Table 1). However, a significantly higher percentage of patients in the hematoma group had joint deformity (100 vs. $66.7 \%, P<0.001$ ), and accordingly, the operation time was significantly longer for these patients $(83.9 \pm 3.1$ vs. $70.5 \pm 4.2 \mathrm{~min}, P<0.001)$ than for patients in the non-hematoma group.

\section{Comparison of coagulation functions}

No significant differences were observed in APTT, PT, TT, and FIB between patients in the hematoma and non-hematoma groups preoperatively $(P>0.05)$. In comparison to preoperative values, APTT was significantly increased in the hematoma group on postoperative days 1,3 , and 7 and in the non-hematoma group on postoperative days 3 and $7(P<0.05$, Fig. 1$)$. In addition, the hematoma group showed a significantly higher APTT than the non-hematoma group on postoperative days 1,3 , and $7(P<0.05)$. Also, in comparison to preoperative values, PT was significantly increased in the hematoma group on postoperative days $1,3,7$, and $14(P<0.05)$ but not in the non-hematoma group. In addition, FIB was significantly increased in the non-hematoma group on postoperative days 3,7 , and 14 but only on postoperative day 7 in the hematoma group $(P<0.05)$. 
Table 1 Comparison of baseline data between patients in the hematoma group and the non-hematoma group

\begin{tabular}{llll}
\hline & $\begin{array}{l}\text { Hematoma } \\
\text { group }(n=32)\end{array}$ & $\begin{array}{l}\text { Non-hematoma } \\
\text { group }(n=114)\end{array}$ & $P$ value \\
\hline Sex (male/female) & $11 / 21$ & $40 / 74$ & 0.94 \\
Age (years) & $70.6 \pm 4.1$ & $70.9 \pm 4.7$ & 0.932 \\
Diabetes, $n(\%)$ & $12(37.5 \%)$ & $28(24.6 \%)$ & 0.147 \\
Hypertension, $n(\%)$ & $25(78.1 \%)$ & $71(62.3 \%)$ & 0.095 \\
BMl (kg/m $\left.{ }^{2}\right)$ & $25 \pm 2.2$ & $26 \pm 2.4$ & 0.061 \\
Joint deformity, $n(\%)$ & $32(100 \%)$ & $76(66.7 \%)$ & $<0.001$ \\
Operation time (min) & $83.9 \pm 3.1$ & $70.5 \pm 4.2$ & $<0.001$ \\
\hline
\end{tabular}

\section{Comparison of hemorheological parameters}

The whole blood high shear rates, whole blood low shear rates, plasma viscosities, and hematocrit levels did not differ significantly between the hematoma and non-hematoma groups at baseline or from postoperative days $1-14 \quad(P>0.05)$. However, the hematocrit level was significantly decreased on postoperative days 1,3 , and 7 in the hematoma group compared with the baseline value $(P<0.05$, Fig. 2$)$.

\section{Discussion}

The specific mechanisms underlying the formation of ecchymoses after TKA remain unclear. However, our study showed that patients who experienced ecchymoses had significantly prolonged APTTs and PTs as well as significantly decreased hematocrit levels after TKA in comparison with baseline values. These findings may help clinicians identify patients at increased risk for hematoma formation after TKA.

In our study, preoperative coagulation functions did not differ significantly between patients with and without postoperative hematoma. Therefore, we are unable to predict the occurrence of hematoma based on preoperative coagulation functions. However, among patients with a hematoma, APTT was significantly prolonged to beyond $10 \mathrm{~s}$ on postoperative day 1 in comparison with baseline data. On the contrary, APTT in the non-hematoma group on postoperative day 1 was not significantly different from the baseline value. These results suggest that a prolonged APTT on postoperative day 1 may be predictive of hematoma formation. In addition, blood tests were performed on postoperative day 1 before the administration of apixaban. Therefore, the prolonged APTT was not caused by apixaban.
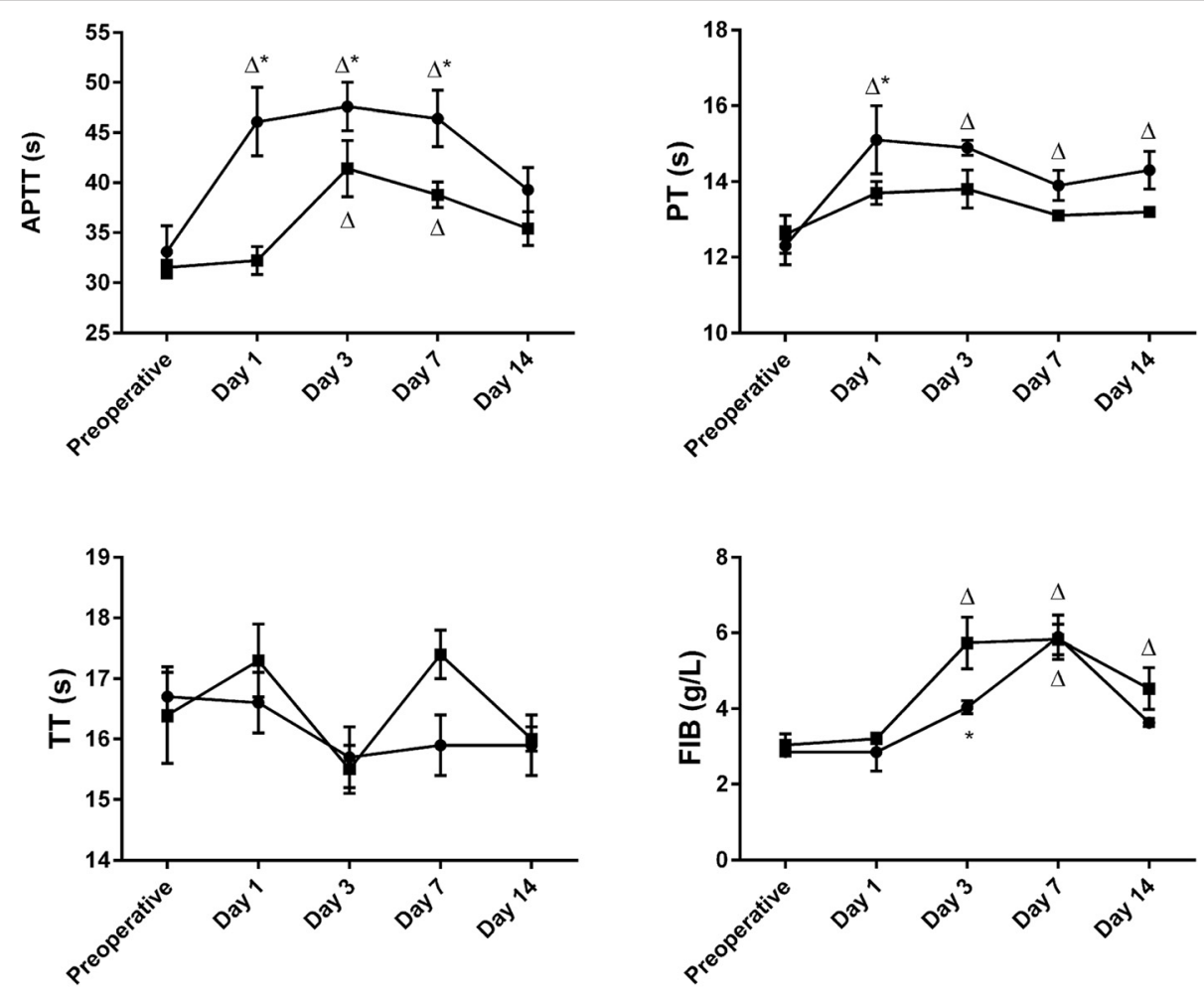

$\rightarrow$ Non-ecchymosis group

$\rightarrow$ Ecchymosis group

Fig. 1 Changes in APTT, PT, T, and FIB in the hematoma group and the non-hematoma group. Triangle $P<0.05$ vs. preoperative values, asterisk $P<0.05$ vs. the other group at the same time point 

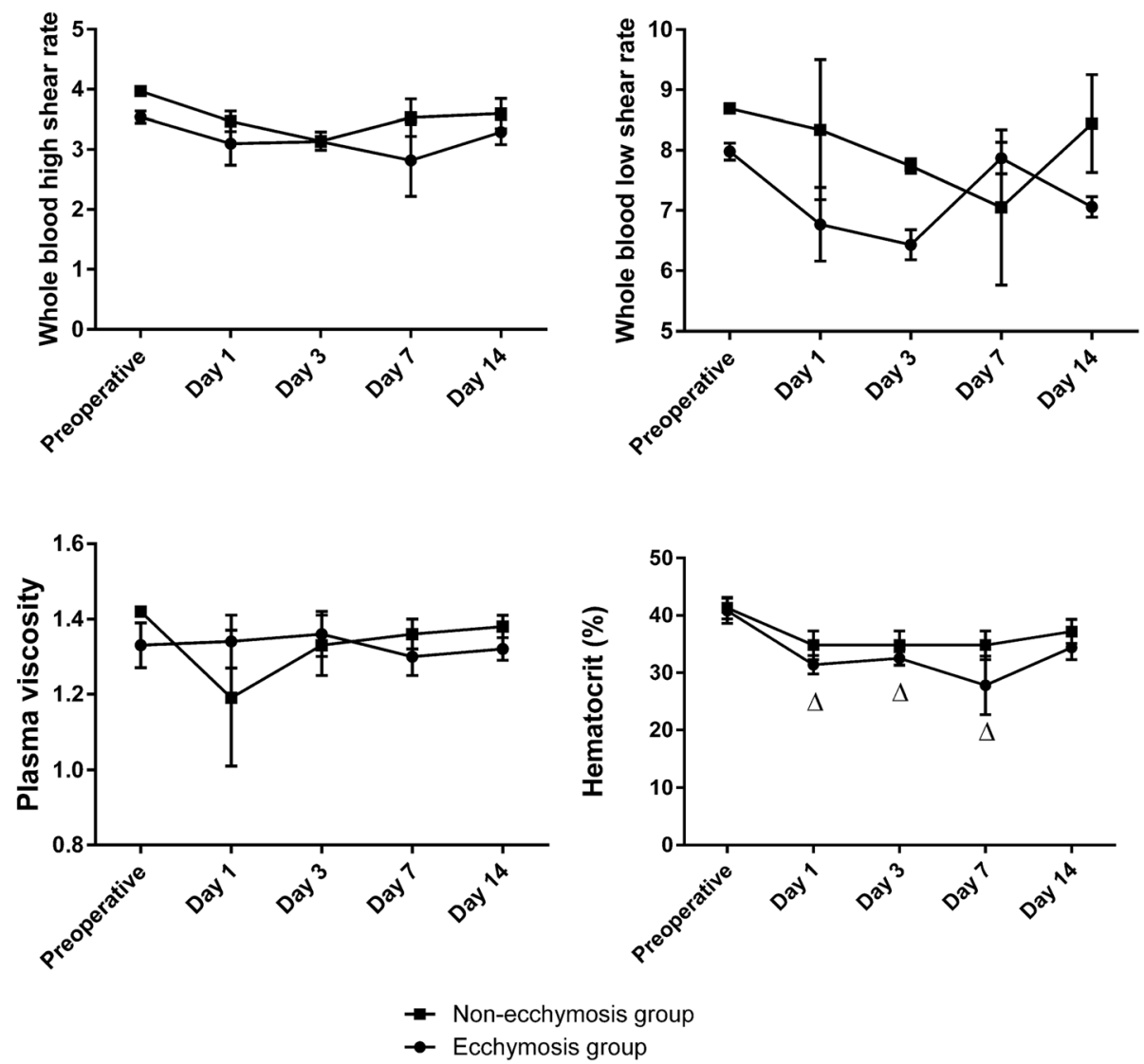

Fig. 2 Changes in whole blood high shear rate, whole blood low shear rate, plasma viscosity, and hematocrit in the hematoma group and the non-hematoma group. Triangle $P<0.05$ vs. preoperative values, asterisk $P<0.05$ vs. the other group at the same time point

Similar patterns were also observed for PT, which was significantly prolonged during the postoperative period in the hematoma group but not in the non-hematoma group. Thus, our results indicate that, together, prolonged APTT and PT on postoperative day 1 can be used to predict hematoma formation after TKA.

Hemorheological parameters reflect the properties of blood flow, with increased hemorheological parameters indicating higher blood viscosity and greater risks of thrombosis formation [14]. Blood viscosity is determined by both the whole blood viscosity and plasma viscosity. An increase in whole blood viscosity with a high shear rate can lead to decreased erythrocyte deformability, whereas an increase in whole blood viscosity with a low shear rate can lead to increased erythrocyte aggregation [15]. Our study found that the whole blood high shear rate, whole blood low shear rate, plasma viscosity, and hematocrit level did not differ significantly between patients in the hematoma and non-hematoma groups at baseline and from postoperative days $1-14$. These results suggest that the erythrocyte deformability and aggregation were not significantly altered by the TKA procedure. We speculate that the use of apixaban helped to maintain the blood viscosity in the patients included in this study. A decreased hematocrit level is a sign of anemia or hemodilution [16], and in our study, hematocrit levels were significantly decreased in the hematoma group on postoperative days 1,3 , and 7 compared to the baseline value. In contrast, hematocrit levels in the non-hematoma group did not change significantly. We speculate that the hematoma group had a higher incidence of hemodilution, leading to decreased levels of coagulant factors and increased bleeding risk.

\section{Conclusions}

In conclusion, prolonged APTT and PT on the first day after TKA together with a decreased hematocrit level may indicate an increased risk of hematoma formation. Postoperative use of apixaban may promote the formation of ecchymoses but is not a major contributing factor. Infusion of plasma or coagulant factors might be an effective prophylaxis measure to prevent hematoma formation after TKA.

\section{Competing interests}

No benefits in any form have been or will be received from a commercial party related directly or indirectly to the subject of this manuscript. 


\section{Authors' contributions}

$\mathrm{NL}, \mathrm{SL}, \mathrm{CH}$, and ZZ conceived and designed the experiments. NL, SL, JL, WW, and $\mathrm{DH}$ performed the experiments. $\mathrm{WW}$ and $\mathrm{DH}$ analyzed the data. SL wrote the paper. SL involved in the design of study analysis and revision of the manuscript. All authors read and approved the final manuscript.

\section{Acknowledgements}

This project was supported by a grant from the National Natural Science Foundation of China (No. 81472089), Guangzhou Provincial Science and Technology Project of China (2014Y2-00084), and the Science and Technology Development Fund of Macau.

\section{Author details}

'The First Affiliated Hospital, Jinan University, Guangzhou 510632, China. ${ }^{2}$ Institute of Orthopaedic Disease Research, Jinan University, Guangzhou 510632, China. ${ }^{3}$ Macau Medical Science \& Technology Research Association, Macau, China.

Received: 28 December 2015 Accepted: 16 March 2016

\section{Published online: 24 March 2016}

\section{References}

1. He ML, et al. Continuous passive motion for preventing venous thromboembolism after total knee arthroplasty. Cochrane Database Syst Rev. 2014;7:CD008207.

2. Lieberman JR, Pensak MJ. Prevention of venous thromboembolic disease after total hip and knee arthroplasty. J Bone Joint Surg Am. 2013;95(19):1801-11.

3. Idestrup C, et al. The incidence of hematoma formation in patients with continuous femoral catheters following total knee arthroplasty while receiving rivaroxaban as thromboprophylaxis: an observational study. Reg Anesth Pain Med. 2014;39(5):414-7.

4. Kang $Y$, et al. Blood transfusion and drainage catheter clamping are associated with haematoma formation at the surgical site after total knee arthroplasty: an analysis of 102 unilateral cases. Eur J Orthop Surg Traumatol. 2013;23(2):219-24

5. Liu XH, et al. The effect of drainage tube on bleeding and prognosis after total knee arthroplasty: a prospective cohort study. J Orthop Surg Res. 2014;9:27.

6. Esler CN, Blakeway C, Fiddian NJ. The use of a closed-suction drain in total knee arthroplasty. A prospective, randomised study. J Bone Joint Surg Br. 2003:85(2):215-7.

7. Seo ES et al. Subcutaneous versus intraarticular indwelling closed suction drainage after TKA: a randomized controlled trial. Clin Orthop Relat Res. 2010;468(8):2168-76

8. Fan $Y$, et al. Drainage does not promote post-operative rehabilitation after bilateral total knee arthroplasties compared with nondrainage. Chin Med Sci J. 2013;28(4):206-10

9. Li B, et al. The effect of tourniquet use on hidden blood loss in total knee arthroplasty. Int Orthop. 2009;33(5):1263-8.

10. Zou Y, et al. Administering aspirin, rivaroxaban and low-molecular-weight heparin to prevent deep venous thrombosis after total knee arthroplasty. Blood Coagul Fibrinolysis. 2014;25(7):660-4.

11. Li B, et al. The effect of knee position on blood loss and range of motion following total knee arthroplasty. Knee Surg Sports Traumatol Arthrosc. 2012;20(3):594-9.

12. Ma T, et al. Effect of flexion/extension splintage post total knee arthroplasty on blood loss and range of motion — a randomised controlled trial. Knee. 2008;15(1):15-9.

13. Hochberg MC, et al. Guidelines for the medical management of osteoarthritis. Part II. Osteoarthritis of the knee. American College of Rheumatology. Arthritis Rheum. 1995;38(11):1541-6.

14. Lee KW, Blann AD, Lip GY. High pulse pressure and nondipping circadian blood pressure in patients with coronary artery disease: relationship to thrombogenesis and endothelial damage/dysfunction. Am J Hypertens. 2005;18(1):104-15.

15. Hamlin SK, Benedik PS. Basic concepts of hemorheology in microvascular hemodynamics. Crit Care Nurs Clin North Am. 2014;26(3):337-44.

16. Van PY, et al. Blood volume analysis can distinguish true anemia from hemodilution in critically ill patients. J Trauma. 2011;70(3):646-51.

\section{Submit your next manuscript to BioMed Central and we will help you at every step:}

- We accept pre-submission inquiries

- Our selector tool helps you to find the most relevant journal

- We provide round the clock customer support

- Convenient online submission

- Thorough peer review

- Inclusion in PubMed and all major indexing services

- Maximum visibility for your research

Submit your manuscript at www.biomedcentral.com/submit 